\title{
Autophagy in Adipose Tissue
}

\author{
Nitzan Maixner $^{a} \quad J_{u l i a ~ K o v s a n}{ }^{a} \quad$ Ilana Harman-Boehm ${ }^{b}$ Matthias Blüher ${ }^{c}$ \\ Nava Bashan ${ }^{a} \quad$ Assaf Rudich $^{a}{ }^{d}$ \\ ${ }^{a}$ Department of Clinical Biochemistry and Pharmacology, Faculty of Health Sciences, \\ Ben-Gurion University of the Negev, ${ }^{b}$ Internal Medicine $C$ and the Diabetes Clinic, Soroka \\ Academic Medical Center, Beer-Sheva, Israel, 'Department of Medicine, University of \\ Leipzig, Leipzig, Germany, ${ }^{d}$ The National Institute of Biotechnology in the Negev, \\ Ben-Gurion University of the Negev, Beer-Sheva, Israel
}

The possible involvement of the evolutionarily conserved process of autophagy (selfeating) in the pathogenesis of human diseases has been increasingly appreciated in recent years. However, at present, many fundamental questions remain to be answered before autophagy can be considered as a therapeutic target: The biological consequences of activating or inhibiting the process are very much cell type-specific and overall biological context-dependent. Consequently, the same manipulation can yield opposing effects, such as enhanced cell survival versus cellular death, or augmented versus attenuated cellular stress responses. In this brief perspective editorial, we highlight the current knowledge on the role of autophagy in adipose tissue (AT). We first address the putative role of autophagy in AT development, and then discuss how AT autophagy is altered in obesity as well as the possible causes for such alteration. Next, we raise outstanding questions on the role altered AT autophagy might play in the context of obesity, and the potential to manipulate adipose autophagy for therapeutic purposes.

\section{Introduction}

Cellular homeostasis involves continuous coordinated biosynthesis and degradation processes that respond to changing environmental conditions by adjusting cellular content to the changing physiological needs [1]. Within this context, degradation processes including the ubiquitin-proteasome pathway and autophagy are particularly essential. Autophagy is a process by which intracellular components are targeted for lysosomal degradation by a highly regulated process of vesicle formation and fusion (reviewed in detail elsewhere

Assaf Rudich, MD, Ph.

Department of Clinical Biochemistry and Pharmacology

Faculty of Health Sciences, The National Institute of Biotechnology in the Negev

Ben-Gurion University of the Negev, 84103 Beer-Sheva (Israel)

rudich@bgu.ac.il 


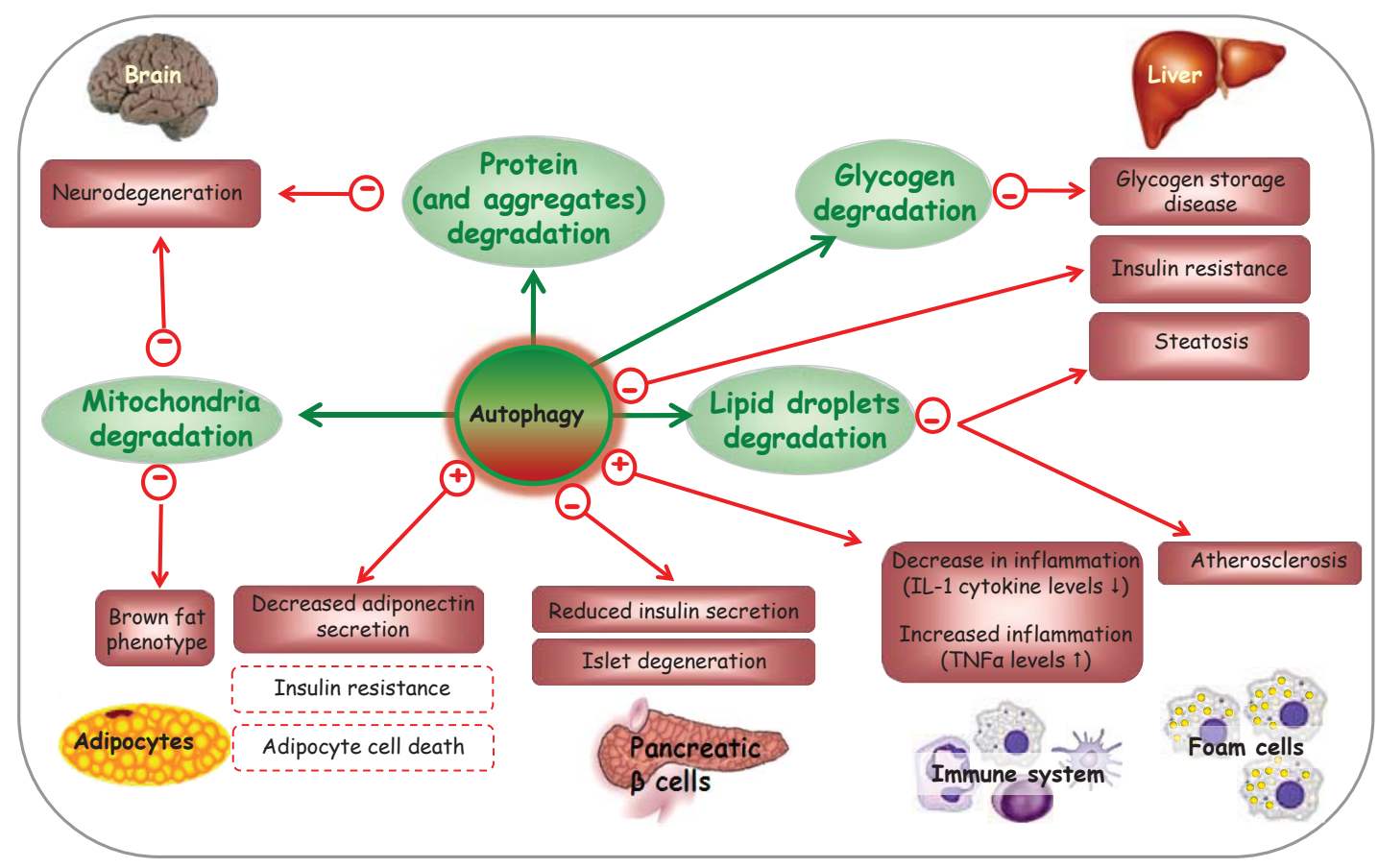

Fig. 1. Autophagy in healthy metabolism and pathophysiology.

Autophagy is a crucial house-keeping mechanism and as such it is involved in a variety of cellular pathways. Hence, alternations in autophagy levels (increase/decrease) may have major functional consequences and ultimately lead to disorders. Green arrows and shapes represent autophagy-mediated cellular processes. The +/- signs at the head of the red arrows indicate whether an autophagy is increased or inhibited, and red shapes denote the functional outcome of such respective alternation, in the relevant tissue. The dashed red shapes designate putative metabolic outcomes.

[1-3]). Autophagy is strongly induced in response to nutrient starvation conditions and its products (amino acids, fatty acids, monosaccharides) used for energy supply and for the synthesis of components essential for survival [1]. Defects in autophagy cause a significant reduction in the intracellular amino acid pool, subsequently leading to an inability of the cell to synthesize proteins that are required for survival [4], hence potentially resulting in cellular death. In addition, autophagy is important for cellular housekeeping because it eliminates unnecessary, damaged, and/or potentially harmful cellular products and organelles $[1,2]$. Given these fundamental functions, it is not surprising that autophagy has been studied in the context of basic biological processes, including aging [5], cellular development [3] and cellular defense against pathogens [6], and is implicated in the pathogenesis of a growing number of human diseases.

Autophagy recently received increasing interest as it emerged as a key regulator of different aspects of cancer biology [7] and energy metabolism [2, 8, 9]. In energy and metabolic regulation, autophagy is thought to contribute to carbohydrate (glycogen) and protein degradation $[8,10]$ and may be important for the regulation of lipid metabolism $[9,11]$. More recently, autophagy has been implicated in the pathophysiology of obesity and its related metabolic diseases [7-9] (fig. 1). Studies in mice with an AT-specific disruption of the critical autophagy gene, autophagy-related 7 (Atg7), revealed that autophagy contributes to the regulation of AT mass and the balance between white and brown fat $[12,13]$. Importantly, Atg7 knockout mice are lean, insulin-sensitive, have enhanced metabolic rate, and are resistant to the development of obesity $[7,8]$. In human studies, we and others found that 
autophagy is up-regulated in AT of obese and/or type 2 diabetic individuals, predominantly in the omental fat depot $[14,15]$. Here, we discuss the current state-of-the-art on the role of autophagy in the regulation of AT development as well as causes, mechanisms, and consequences of increased AT autophagy in obesity.

\section{Autophagy and Adipose Tissue Development}

It is well established that fully functional autophagy is essential during development from early embryogenesis, over stem cell differentiation into various specialized cell types up to the adaptation and survival of neonates [16]. First evidence for a role of autophagy specifically in AT development was noted by assessing the phenotype of Atg5-/- mice in which whole-body autophagy is defective [17]. The knockout mice die within the first day after birth, highlighting the importance of autophagy in normal development and/or in adaptation to the early neonatal period environment. Yet, post mortem analysis of their subcutaneous tissues revealed drastically reduced perilipin-A-positive adipocytes in comparison to wild-type counterparts. Similar findings were evident in both embryos and neonates, suggesting a potential role for Atg5 and/or autophagy in embryonal development of AT. However, deducing the direct role of a certain gene product in a specific tissue from a whole-body knockout model is somewhat precarious, as apparent changes may result indirectly from primary effects in other tissues. To more specifically delineate the role of autophagy in AT development, two complementary approaches have been utilized:

i) An in-vitro approach: Observing the impact of autophagy genes knockout on preadipocytes in vitro, thereby isolating cell-autonomous mechanisms from outer influences;

ii) An in-vivo approach using tissue-specific knockout techniques, which results in disrupted autophagy in AT only in the context of an otherwise normal body.

Using either the 3T3-L1 cell line or mouse embryonic fibroblasts (MEFs) revealed that normal pre-adipocyte adipogenesis is associated with increased autophagic activity (measured by both number of autophagosomes and decrease in autophagy-degraded proteins such as p62) [17]. MEFs from Atg5-/- cells fail, upon inducing adipogenesis, to adopt spherical morphology. Although micro lipid-droplets do begin to form they remain fewer and smaller than in wild-type cells and subsequently fail to fuse and form a large single droplet. The amount of accumulated triglycerides in the cytoplasm, a main hallmark of adipogenesis, remains lower in knockout than in wild-type cells at all times during cell differentiation [17]. Consistently, inefficient adipogenesis in Atg5-/- cells is also evident by decreased induction of ppar $\gamma$ and cebp $\alpha$ mRNA and their protein products in comparison to wild-type cells. This difference is further accentuated when assessing later-phase adipogenesis genes/markers, such as Fabp4, perilipin, fatty acid synthase, GLUT4, and SCD1. Finally, the knockout cells exhibit higher levels of apoptosis; while normal pre-adipocytes present less apoptosis as differentiation advances, in knockout cells apoptosis increases during adipogenesis. It seems that knockout cells can initiate the differentiation process, but die as they fail to complete it. Essentially similar results to those observed with Atg5knockout cells were also described in 3T3-L1 or MEFs in which Atg7 was targeted [12,13], or when autophagy was inhibited pharmacologically using chloroquine [17] or 3-methyladenine [12]. Collectively, autophagy appears essential for completion of adipogenesis, acting as a survival mechanism of cells during the differentiation process.

As an in vivo approach, cre-lox technology was used to generate mice with defective autophagy specifically in (pre-)adipocytes $[12,13]$. Adipocyte-specific Atg7-knockout mice exhibited adipocytes with features more characteristic of brown fat than of white AT, being heterogeneous, smaller, containing multiple small lipid droplets and a significant amount 
of cytoplasm, in contrast to the typical homogenous unilocular cells of wild-type mice. Cells also exhibited a markedly increased number of mitochondria $[12,13,18]$. At the whole body level white fat depots (gonadal, scapular) were about $20 \%$ smaller, with whole body weight being consistently lower. This was witnessed despite comparable food intake in the knockout mice as compared to wild-type mice (or even increased if normalized to body weight), suggesting reduced efficiency in energy storage and/or higher energy expenditure [13]. When quantified for gene expression, AT of the knockout mice did not exhibit any pattern change in the expression of major adipocyte-related genes (including ppar $\gamma$, fabp4, cebpa, gpam). This may seem contradictory to the in vitro studies described above, and a potential explanation may lie in the exact stage at which autophagy was disrupted (an aP2-Cre system may inactivate autophagy at later stages in adipogenesis than when Atg5 is knocked out already at the pre-adipocyte stage). In addition, studies were inconsistent as to the extent that the altered AT of adipose-specific knockout mice express characteristic brown fat genes $[12,13]$. Nevertheless, the rate of beta-oxidation of fatty acid in knockout mice was evidently higher while hormone-induced free fatty acid release was lower, suggesting preferred fatty acid channeling to oxidation $[12,13,18]$. Autophagydefective AT resulted in improved insulin sensitivity, lower triglyceride levels, non-esterifed fatty acids and cholesterol, markedly lower levels of leptin but no evident change in adiponectin levels. Another remarkable feature of the adipose-specific Atg7-knockout mice was an apparent resistance to high-fat diet: no significant weight gain was seen in high-fat diet knockout mice in comparison to low-fat diet knockout mice, and this was associated with increased activity thermogenesis and total energy expenditure [13]. Finally, it is of interest that inconsistent with the generally improved phenotype of the adipose-specific knockout mice, they presented with higher macrophage infiltration in white AT upon high-fat feeding compared to wild-type counterparts, suggesting that intact AT autophagy may limit high-fat diet-induced AT inflammation [12]. Moreover, knockout mice seemed to be infertile and presented higher spontaneous death rates [13]. In conclusion, AT-specific Atg7knockout mice in which autophagy was interrupted during adipogenesis were visibly slimmer, more active, having improved metabolic measures, but were not necessarily 'globally' healthier.

\section{Altered Adipose Tissue Autophagy in Obesity}

While the previous section clearly demonstrates an involvement of autophagy in AT development, it does not reveal the role(s) of autophagy in the physiology and pathophysiology of mature AT or adipocytes. Adipocytes have a limited cytoplasmic volume, and cytosolic organelles are, in general, rather scarce and difficult to image. Therefore, autophagosomes have only rarely been reported in mature adipocytes [19]. Nonetheless, particular attention has been recently given to the potential contribution of autophagy to AT dysfunction and obesity.

We have recently investigated whether autophagy was altered in ATs of lean and obese humans with a wide range of glucose tolerance and insulin sensitivity [14]. Using complementary approaches, we have provided evidence to support the notion that autophagy is elevated in AT in obesity. This conclusion was based on four key findings:

i) Increased protein content of key autophagy genes - $\operatorname{Atg} 5, L C 3$ and the $\operatorname{Atg} 12-\operatorname{Atg} 5$ complex.

ii) While the above is potentially indicative of activated autophagy, since some autophagy proteins are degraded in the autophagic process, their increased expression level may in fact indicate the opposite, i.e, inhibited autophagy [20]. We therefore used dynamic measures of 
'autophagic flux', in which AT explants were incubated in the absence or presence of autophagy inhibitors. The degree of increase in protein content of autophagy genes, and/or in the number of autophagosomes per cell, which are induced by the inhibitor, indicates the level of activation of the 'autophagic flux'. Indeed, AT from obese patients exhibited a greater increase in the protein content of LC3II, and of p62 and NBR1 - proteins largely degraded by autophagy, upon incubation with autophagy inhibitors.

iii) A larger number of autophagosomes accumulated in these tissues, identified by indirect immunofluorescence detection of the autophagosome marker LC3. These findings described in ii) and iii) indicate increased autophagic flux in AT of obese persons.

iv) Finally, mRNA levels of Atg5 and LC3 were also increased in AT of obese persons. This is significant because it demonstrates regulation of autophagy at the transcriptional level, which may be less ambiguous as an indicator of the activity of the autophagy process.

It is noteworthy that the increase in autophagy was more evident in omental fat than in abdominal subcutaneous fat, and particularly in obese patients with predominantly intraabdominal (central) adiposity. This may be noteworthy given the known strong association between visceral fat and obesity-associated cardiometabolic morbidity. In addition, multiple differences between subcutaneous and visceral fat are known [21] and may provide important clues as to causes and/or consequences of augmented autophagy in AT in obesity. It is, however, important to stress that both the elevated expression of autophagy genes and the higher number of autophagosomes observed, occur both in adipocytes and cells of the stroma-vascular fraction comprising AT. This finding of increased autophagy in adipocytes is consistent with other studies in adipocytes from type 2 diabetic patients $[15,19]$. Yet, another report suggested decreased, rather than increased, adipocyte and AT autophagy in high-fat fed mice [22], a finding we were unable to reproduce (see below and fig. 2).

\section{Causes of Increased Adipose Tissue Autophagy in Obesity}

While acknowledging that increased AT autophagy in obesity may not be the universal finding in the existing literature, one of the major tasks that arises is defining the cause of such phenomenon. Given the impact of the obese state on AT biology, the potential reasons for activated autophagy in this tissue are numerous. The microenvironment of AT is markedly altered by obesity, becoming hypoxic, with increased oxidative stress, inflammatory mediators, and induction of endoplasmic reticulum stress [23-27]. All of these can potentially induce autophagy [28-32], either as a cell survival mechanism or, if cells fail to adequately cope with stress-induced damages, as a cell death process. An additional interesting possibility arises from the demonstration that expression of autophagy genes exhibits a strong diurnal rhythm and may be regulated by biological clock genes [33, 34]. Given the increasingly appreciated role of disrupted biological clocks in metabolic diseases $[35,36]$ and early reports that clock genes may be altered in AT in obesity [37,38], it is tempting to propose disturbed clock gene regulation as a putative cause of, or at least a significant contributor to, altered autophagy in AT. In addition, in many obese individuals AT becomes insulinresistant. Since insulin is a major physiological inhibitor of autophagy, it is plausible that increased autophagy is a manifestation of AT insulin resistance. In particular, the attenuation of the insulin-mediated PI3-kinase pathway and its downstream effector mTOR was proposed as a molecular mechanism for increased AT autophagy in type 2 diabetes [19]. Moreover, by comparing 30 matched pairs of severely obese persons who either remained insulin-sensitive or developed insulin resistance but were otherwise free of cardiometabolic manifestations of obesity, we demonstrated that expression of autophagy genes was only elevated in those who were insulin-resistant [14]. 
Fig. 2. Reciprocal obesity-induced regulation of the expression of autophagy genes in liver and AT.

C57Bl-6 mice were fed either normal chow diet or a high fat $(60 \%)$ diet for 16 weeks to induce obesity, after which epididymal fat pads and livers were collected. A Tissue lysates were prepared from whole AT or liver, and subjected to western blot analysis to detect Atg5 and Atg12-Atg5 complex. Shown are representative blots (vertical white lines indicate splicing of the same membrane image to increase the clarity of the presentation), and densitometry analyses of at least 5 mice per group. An arbitrary value of 1 was given to the mean intensity of the bands from the lean (chow-fed) mice. ${ }^{* *} \mathrm{p}<0.01$ compared to the lean mice. B mRNA was extracted and cDNA generated by reverse transcription, and subjected to quantitative real-time PCR analysis using specific TaqMan probes for the indicated autophagy genes. Results are expressed as the percent change from the mean expression in the chow fed lean mice.

\section{A}

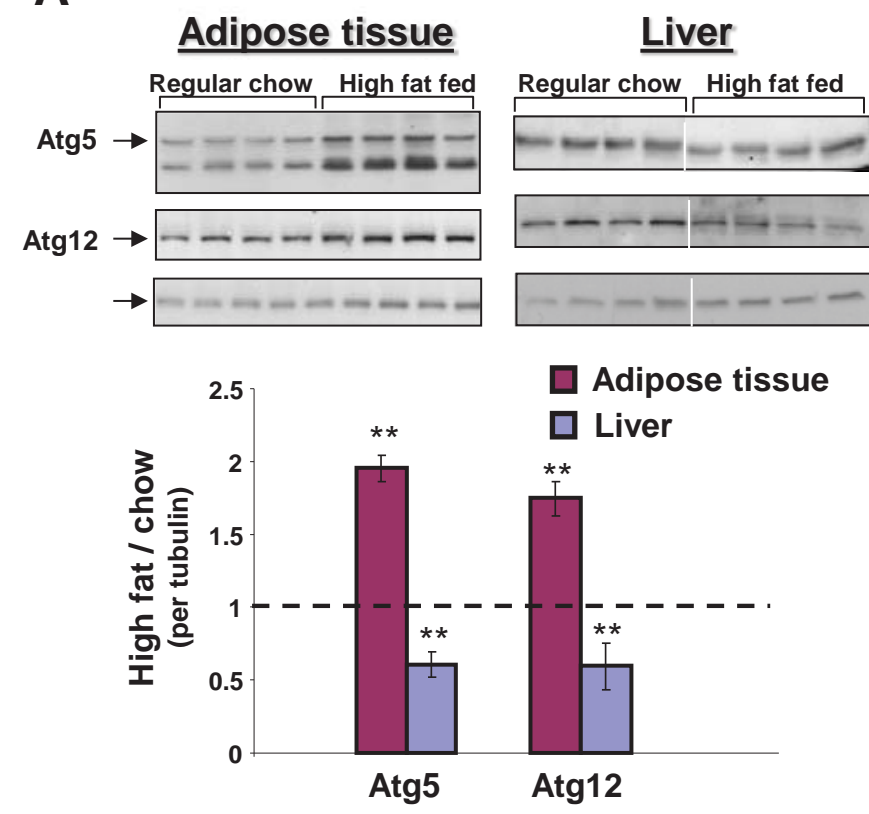

B

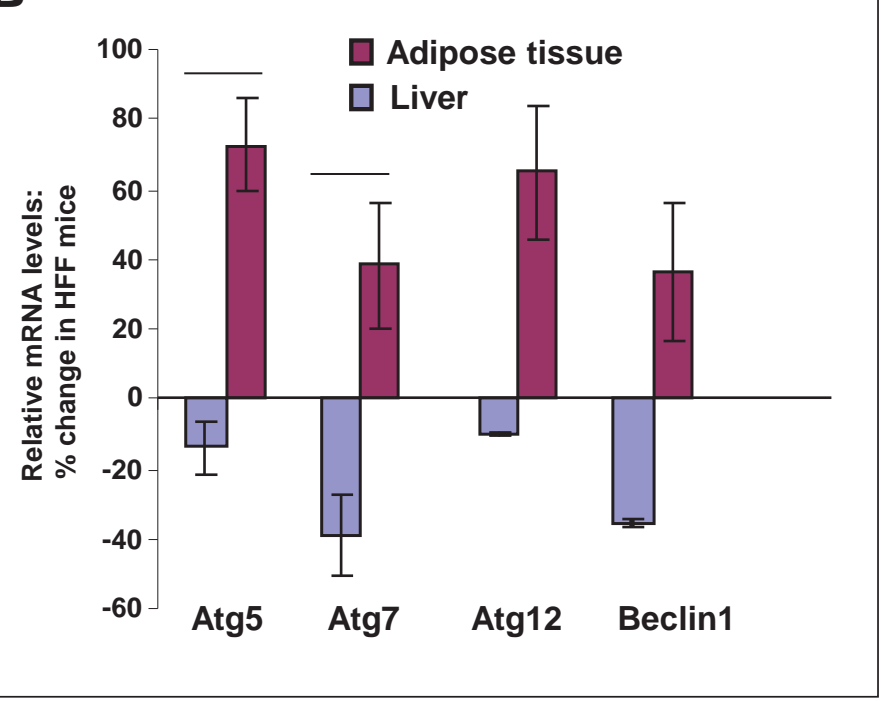

While providing multiple plausible explanations for the induction of autophagy in AT in obesity, it should be noted that the above mentioned stresses, as well as the insulin resistance that associates with them, also develop in other tissues in obesity, e.g., in the liver. Intriguingly, while human studies have suggested increased autophagy in AT, studies in mice suggested that in the liver autophagy is attenuated in obesity [39]. It is therefore possible that the apparent discrepancy can arise from either a 'human-mouse difference' in the response to obesity or a 'fat-liver tissue difference'. To address this question, we have assessed the expression of autophagy genes at the protein and mRNA level in AT and in the liver of the same lean or nutritionally obese mice. As shown in figure 2A, obese (high-fat fed) mice had higher protein levels of Atg5 and Atg12-Atg5 complex in AT than lean mice, but 
lower levels in the liver. Correspondingly, mRNA levels of several autophagy genes were reciprocally regulated in the two tissues by obesity figure $2 \mathrm{~B}$.

Collectively, autophagy appears to be elevated in AT and in adipocytes in obesity both in humans and in mouse models of nutritional obesity. In humans this may be more pronounced in visceral AT than in abdominal subcutaneous fat, particularly when obese persons exhibit more pathogenic forms of fat distribution (central adiposity). Finally, activated autophagy may be a unique feature of AT in the obese state as autophagy was shown to be inhibited in other tissues such as the liver.

\section{What Are the Functional Consequences of Increased Adipose Tissue Autophagy in Obesity?}

Before discussing specific functional outcomes suggested or implied for increased autophagy in AT, it is important to note that various factors proposed to induce AT autophagy (such as insulin resistance, endoplasmic reticulum stress, inflammation, etc.), may in fact appear here as the consequence of elevated autophagy. While seeming contradictory, these two options of a factor being a cause or consequence of the same process are not mutually exclusive. In fact, in pathophysiology vicious cycles or positive feedback loops are very common, representing a situation in which a factor could constitute both the cause and the consequence.

Secondly, it is worth noting that when viewed as a house-keeping process, inhibited autophagy is the more frequent alteration of autophagy that is implicated in pathophysiology. This is the case in aging-related processes [40], neurodegeneration [41], and, as mentioned above, as a cause for hepatic insulin resistance and steatosis in obesity $[9,39]$. According to this view, elevated autophagy of AT may be viewed as an attempt to overcome the consequences of AT stresses. If so, it represents an adaptive response protecting against even more severe dysfunction. However, autophagy can induce diverse and occasionally opposing effects, acting in a cell type-specific and biological context-dependent manner. It is therefore possible that increased autophagy represents an integral part of the stress response and contributes to, rather than protects against, AT dysfunction.

\section{Insulin Resistance}

Autophagy activated in adipocytes in response to inducers of endoplasmic reticulum stress was shown to degrade the insulin receptor [42]. When autophagy was inhibited with 3-methyladenine, thapsigargin-induced insulin receptor degradation was largely prevented. However, this rescue of insulin receptor content did not result in higher insulin signal transmission to Akt or towards the stimulation of glucose uptake. Thus, while insulin resistance is a likely cause of increased autophagy in adipocytes [19] and autophagy may contribute to lower insulin receptor protein content in AT in obesity [42], it remains unclear whether elevated autophagy contributes to metabolic insulin resistance. Clinically, autophagy genes were more highly expressed in omental fat of the obese insulin-resistant patients than in their age-, sex- and BMI-matched insulin-sensitive counterparts [14]. While the significance of this finding is in the proposition that increased autophagy develops along with insulin resistance (and precedes obesity-associated morbidities), it cannot delineate whether insulin resistance is the cause and/or the result of, or is even unrelated to, the elevated AT autophagy.

\section{Adipocyte Cell Death}

In obesity, cell death of hypertrophied adipocytes has been suggested to occur and to exhibit morphological features inconsistent with classical apoptotic or necrotic cell death 
[43]. While increased autophagy may contribute to cell survival, it has also been shown, in certain circumstances, to constitutean alternative or complementaryprocess of 'programmed' cell death [44]. It would therefore seem important to determine whether elevated adipocyte autophagy contributes to enhanced adipocyte cell death in obesity. Moreover, a 'merged' autophagic-phagocytic process may be important for degradation of dead cell debris within macrophages [45]. Thus, increased autophagy of AT may relate to both augmented adipocyte cell death and to the increased requirement for removal of adipose cellular debris.

\section{Adipose Tissue Inflammation}

Proinflammatory cytokines have largely been shown to induce autophagy and antiinflammatory cytokines to attenuate the process [28], suggesting that increased proinflammatory cy tokine production by AT in obesity may contribute to the increased AT autophagy. However, can elevated autophagy regulate AT inflammation? On the one hand, elevated autophagy can limit inflammation, thereby providing an inhibitory feedback loop on inflammatory tone. One example is the inhibitory effect of autophagy on the IL-1 family of proinflammatory cytokines, acting at the level of secretion, transcription, inflammasomemediated protein processing, and degradation of IL-1 $\beta$-containing vesicles $[28,46,47]$. Additionally, autophagy was required for survival of antiinflammatory (M2, i.e., CD206+) monocytes exposed to IL-6 and MCP-1 [48], two cytokines highly expressed in AT in obesity. On the other hand, autophagy may augment inflammatory responses, thereby participating in a feed-forward regulatory loop with inflammation: When basal autophagy was pharmacologically inhibited, lipopolysaccharide induction of TNF- $\alpha$ secretion from human peripheral blood mononuclear cells was markedly attenuated [47]. Moreover, under conditions of elevated autophagic flux, cells may become hypersensitive to additional inflammatory signals, responding to such stimuli with augmented IL-1 $\beta$ secretion [49]. Thus, augmented AT autophagy may constitute a major fine-tuning factor in determining the inflammatory tone of AT in obesity.

Noteworthy in this context is also the proposition that autophagy may mediate the link between endoplasmic reticulum stress and reduced adiponectin levels in obesity [50,51]. Most direct evidence stems from studies in 3T3-L1 adipocytes, in which induction of endoplasmic reticulum stress was sufficient to promote decreased adiponectin levels, and this effect was prevented by the autophagy inhibitor 3-methyladenine. Given potential autophagy-unrelated effects of 3-methyladenine, the causative role of autophagy has yet to be substantiated. Nevertheless, given the antiinflammatory properties of adiponectin and its role as an immunomodulatory factor [52], autophagy may modulate the inflammatory tone not only by directly affecting cytokine production and release but also indirectly via adiponectin regulation.

\section{Mitochondrial Mass}

As mentioned in 'Autophagy and Adipose Tissue Development' (see above), when autophagy is inhibited during adipocyte development, adipocytes adopt a more brown adipocyte-like phenotype characterized by abundant mitochondria. Although debated, alterations in mitochondrial mass and/or function have been proposed to contribute to the pathophysiology of insulin resistance and type 2 diabetes, and potentially to obesity. While (macro)autophagy is considered a nonselective process, mitochondrial autophagy (mitophagy) is a selective autophagic process 'subtype'. Assuming that elevated autophagy in AT in obesity also represents enhanced mitochondrial degradation, it is intriguing to speculate that increased autophagy contributes to limiting mitochondrial mass in adipocytes, lowering capacity for mitochondrial oxidation of nutrient, and thus, contributing to increased energy storage capacity and to the pathogenesis of obesity itself. 


\section{AT Lipolysis}

Though arguably and possibly different among the various fat depots, it is generally thought that in obesity basal adipocyte lipolysis is increased, whereas stimulated lipolysis is attenuated. Mice with developmental adipocyte-specific defective autophagy exhibited diminished stimulated lipolysis [13]; so, along this line, activated autophagy could be expected to augment lipolysis. Yet, as discussed earlier, the phenotype of the adipocytespecific autophagy-defective mice may represent a consequence of altered adipocyte development in the face of disturbed autophagy and may not reflect the role of autophagy in regulating adipocyte lipolysis in obesity. Also indirectly, interfering with autophagy function in pro-opiomelanocortin (POMC) neurons in the hypothalamus impaired lipolysis and promoted obesity [53]. Thus, how autophagy regulates lipolysis in mature adipocytes is still unclear. Moreover, caution should be taken when using pharmacological inhibitors of autophagy, since a recent study in differentiated 3T3-L1 adipocytes suggested that the autophagy inhibitor 3-methyladenine increased lipolysis, but this could not be attributed to the inhibition of autophagy [54].

Nevertheless, autophagy does seem to participate in lipid droplet degradation in nonadipocytes $[8,9,55]$, raising a putative role for autophagy-regulated lipolysis in non-adipocytes of AT. In this regard, increasing evidence suggests a role for autophagic-lysosomal processes in lipid mobilization from lipid-laden macrophages [56,57]. We have recently identified foam cells in human AT (H. Shapiro, I. Harman-Boehm, M. Blüher, A. Rudich, unpublished data), suggesting that activated autophagy in such cells may contribute to local production of nonesterified fatty acids from non-adipocytes present in AT.

\section{How Far Are We from Targeting Autophagy for Therapeutic Purposes in Obesity?}

The mysteries of autophagy in the development of obesity and obesity-associated metabolic derangement have yet to be unraveled. The literature to date supports involvement of autophagy in opposing pathways: pro- and antiinflammation, pro- and antioxidation, insulin resistance and insulin sensitivity, lipolysis and lipid storage, cell survival and death. These effects may be cell type- and/or tissue-specific, suggesting that autophagy may play a protective role (and thus should ideally be activated) in some tissues, while it contributes to metabolic derangement via multiple mechanisms in other tissues (and thus perhaps should be inhibited). Such tissue or cell type specificity would inevitably require sophisticated means of drug targeting approaches to achieve the wanted effects (and to avoid detrimental consequences) when manipulating autophagy. Therefore, the manipulation of AT autophagy for therapeutic purposes clearly awaits improvement of our understanding of both the various regulatory pathways of autophagy and its contribution to pathophysiology, specifically in AT and, possibly even more challenging, in specific fat depots. Until that time, it would seem premature to target AT autophagy in obesity, an intervention that currently can be expected to potentially prevent metabolic derangement of obesity but also to enhance it. Yet, putting all the quandaries and caveats aside, a glance at the NIH clinical trials registry ( $w w w$. clinicaltrials.gov) reveals some 30 clinical trials examining the effects of drugs affecting autophagy on clinical outcomes, the vast majority of which are designed to assess whether various means of inhibiting autophagy could enhance therapeutic effects of chemotherapy against different types of cancers. The rationale for these studies is based on data that demonstrated that, although autophagy plays a role in preventing tumorogenesis, once malignant transformation occurred activated autophagy supports tumor cell survival [58]. Hence, autophagy inhibitors are studied as potential sensitizers to anticancer therapy and/or as the primary agent [59-61]. These are first examples of translating knowledge gained by studying 
autophagy mechanisms and roles into the clinic. They do, however, provide hope for future ability to employ advanced understanding of the roles of components of autophagy in obesity and target them for therapeutic intervention. Current clinical studies have undoubtedly inspired the search for manipulating autophagy as a therapeutic strategy for treating atherosclerosis [62], cardiovascular disease [63], and neurodegeneration [64], though in all of those it is activation of autophagy that is thought to potentially be beneficial (fig. 1).

In AT it would seem that the first questions that need to be addressed are: i) whether autophagy is indeed activated in obesity; ii) in which AT depots autophagy is activated; and iii) if indeed activated, whether it acts to limit pathogenesis (in which case it should not be inhibited) or contributes to pathophysiology (in which case perhaps it should be downregulated/normalized). As discussed above, the roles autophagy seem to play in AT development and the current findings suggesting that autophagy is activated in AT in obesity would suggest that inhibiting AT autophagy might constitute a strategy to 'brown' AT and increase whole-body energy expenditure in an attempt to combat obesity. Brown adipocyte biogenesis and/or various means of turning white adipocy tes to either brown or brown-like adipocytes are currently a major area of research that should incorporate autophagy as a putative modulator that could be manipulated for therapeutic purposes. A cautionary note is required here, given that many of the studies rely on rodent (particularly mouse) models in which plasticity of AT may be markedly greater than in humans and is based on species-specific neuroendocrinological mechanisms. Nevertheless, if mature human AT can be 'browned' by inhibiting autophagy, this could open an exciting new avenue for normalizing AT in obesity. Future research will undoubtedly reveal whether normalizing lipolysis and/or adiponectin production as well as decreasing adipocyte cell death and AT inflammation are additional beneficial consequences of preventing autophagic hyperactivity in AT in obesity.

\section{Acknowledgements}

We are thankful to Tanya Tarnovscki, for excellent technical assistance. This manuscript and our studies mentioned herein were supported in part by grants by the German-Israel Science Foundation (GIF, grant GIF 995-09 to M.B, N.B. and A.R), The Israeli Ministry of Health (Grant 3/5067 to A.R.), and the Leslie and Susan Gonda (Goldschmid) Center for Diabetes Research and Education. A.R. is chair of the Fraida Foundation in Diabetes Research.

\section{Disclosure Statement}

The authors have nothing to disclose.

\section{References}

- 1 Todde V, Veenhuis M, van der Klei IJ: Autophagy: principles and significance in health and disease. Biochim Biophys Acta 2009;1792:3-13.

- 2 Singh R, Cuervo AM: Autophagy in the cellular energetic balance. Cell Metab 2011;13:495-504.

- 3 Levine B, Klionsky DJ: Development by self-digestion: molecular mechanisms and biological functions of autophagy. Dev Cell 2004;6:463-477.

- 4 Onodera J, Ohsumi Y: Autophagy is required for maintenance of amino acid levels and protein synthesis under nitrogen starvation. J Biol Chem 2005;280:31582-31586.

- 5 Bergamini E: Autophagy: a cell repair mechanism that retards ageing and age-associated diseases and can be intensified pharmacologically. Mol Aspects Med 2006;27:403-410.

- 6 Mizushima N, Levine B, Cuervo AM, Klionsky DJ: Autophagy fights disease through cellular self-digestion. Nature 2008;451:1069-1075.

7 Kimmelman AC: The dynamic nature of autophagy in cancer. Genes Dev 2011;25:1999-2010. 
- 8 Kovsan J, Bashan N, Greenberg A, Rudich A: Potential role of autophagy in modulation of lipid metabolism. Am J Physiol Endocrinol Metab 2010;298:E1-E7.

- 9 Singh R, Kaushik S, Wang Y, Xiang Y, Novak I, Komatsu M, Tanaka K, Cuervo AM, Czaja MJ: Autophagy regulates lipid metabolism. Nature 2009;458:1131-1135.

10 Kotoulas OB, Kalamidas SA, Kondomerkos DJ: Glycogen autophagy in glucose homeostasis. Pathol Res Pract 2006;202:631-638.

11 Shibata M, Yoshimura K, Furuya N, Koike M, Ueno T, Komatsu M, Arai H, Tanaka K, Kominami E, Uchiyama Y: The MAP1-LC3 conjugation system is involved in lipid droplet formation. Biochem Biophys Res Commun 2009;382:419-423.

-12 Singh R, Xiang Y, Wang Y, Baikati K, Cuervo AM, Luu YK, Tang Y, Pessin JE, Schwartz GJ, Czaja MJ: Autophagy regulates adipose mass and differentiation in mice. J Clin Invest 2009;119:3329-3339.

13 Zhang Y, Goldman S, Baerga R, Zhao Y, Komatsu M, Jin S: Adipose-specific deletion of autophagy-related gene 7 (atg7) in mice reveals a role in adipogenesis. Proc Natl Acad Sci U S A 2009;106:19860-19865.

-14 Kovsan J, Bluher M, Tarnovscki T, Kloting N, Kirshtein B, Madar L, Shai I, Golan R, Harman-Boehm I, Schon MR, Greenberg AS, Elazar Z, Bashan N, Rudich A: Altered autophagy in human adipose tissues in obesity. J Clin Endocrinol Metab 2011;96:E268-277.

15 Rodriguez A, Gomez-Ambrosi J, Catalan V, Rotellar F, Valenti V, Silva C, Mugueta C, Pulido MR, Vazquez R, Salvador J, Malagon MM, Colina I, Fruhbeck G: The ghrelin 0-acyltransferase-ghrelin system reduces TNF-alpha-induced apoptosis and autophagy in human visceral adipocytes. Diabetologia 2012;55:3038-3050.

-16 Mizushima N, Levine B: Autophagy in mammalian development and differentiation. Nat Cell Biol 2010;12: 823-830.

17 Baerga R, Zhang Y, Chen PH, Goldman S, Jin S: Targeted deletion of autophagy-related 5 (atg5) impairs adipogenesis in a cellular model and in mice. Autophagy 2009;5:1118-1130.

18 Goldman S, Zhang Y, Jin S: Autophagy and adipogenesis: implications in obesity and type II diabetes. Autophagy 2010;6:179-181.

19 Ost A, Svensson K, Ruishalme I, Brannmark C, Franck N, Krook H, Sandstrom P, Kjolhede P, Stralfors P: Attenuated mTOR signaling and enhanced autophagy in adipocytes from obese patients with type 2 diabetes. Mol Med 2010;16:235-246.

20 Klionsky DJ, Abeliovich H, Agostinis P, Agrawal DK, Aliev G, et al: Guidelines for the use and interpretation of assays for monitoring autophagy in higher eukaryotes. Autophagy 2008;4:151-175.

21 Wajchenberg BL: Subcutaneous and visceral adipose tissue: their relation to the metabolic syndrome. Endocr Rev 2000;21:697-738.

-22 Yoshizaki T, Kusunoki C, Kondo M, Yasuda M, Kume S, Morino K, Sekine O, Ugi S, Uzu T, Nishio Y, Kashiwagi A, Maegawa H: Autophagy regulates inflammation in adipocytes. Biochem Biophys Res Commun 2012;417: 352-357.

23 Trayhurn P, Wang B, Wood IS: Hypoxia in adipose tissue: a basis for the dysregulation of tissue function in obesity? Br J Nutr 2008;100:227-235.

24 Gregor MF, Hotamisligil GS: Thematic review series: adipocyte biology. Adipocyte stress: the endoplasmic reticulum and metabolic disease. J Lipid Res 2007;48:1905-1914.

-25 Maury E, Brichard SM: Adipokine dysregulation, adipose tissue inflammation and metabolic syndrome. Mol Cell Endocrinol 2010;314:1-16.

-26 Sell H, Eckel J: Adipose tissue inflammation: novel insight into the role of macrophages and lymphocytes. Curr Opin Clin Nutr Metab Care 2010;13:366-370.

-27 Furukawa S, Fujita T, Shimabukuro M, Iwaki M, Yamada Y, Nakajima Y, Nakayama O, Makishima M, Matsuda M, Shimomura I: Increased oxidative stress in obesity and its impact on metabolic syndrome. J Clin Invest 2004; $114: 1752-1761$.

28 Harris J:Autophagy and cytokines. Cytokine 2011;56:140-144.

29 Bellot G, Garcia-Medina R, Gounon P, Chiche J, Roux D, Pouyssegur J, Mazure NM: Hypoxia-induced autophagy is mediated through hypoxia-inducible factor induction of BNIP3 and BNIP3L via their BH3 domains. Mol Cell Biol 2009;29:2570-2581.

-30 Rouschop KM, van den Beucken T, Dubois L, Niessen H, Bussink J, Savelkouls K, Keulers T, Mujcic H, Landuyt W, Voncken J. W, Lambin P, van der Kogel AJ, Koritzinsky M, Wouters BG: The unfolded protein response protects human tumor cells during hypoxia through regulation of the autophagy genes MAP1LC3B and ATG5. J Clin Invest 2010;120:127-141.

-31 Eskelinen EL, Saftig P: Autophagy: a lysosomal degradation pathway with a central role in health and disease. Biochim Biophys Acta 2009;1793:664-673.

-32 Wang Y, Singh R, Xiang Y, Czaja MJ: Macroautophagy and chaperone-mediated autophagy are required for hepatocy te resistance to oxidant stress. Hepatology 2010;52:266-277.

-33 Ma D, Li S, Molusky MM, Lin JD: Circadian autophagy rhythm: a link between clock and metabolism? Trends Endocrinol Metab 2012;23:319-325.

-34 Sachdeva UM, Thompson CB: Diurnal rhythms of autophagy: implications for cell biology and human disease. Autophagy 2008;4:581-589.

- 35 Marcheva B, Ramsey KM, Affinati A, Bass J: Clock genes and metabolic disease. J Appl Physiol 2009;107: 1638-1646.

-36 Froy 0: Metabolism and circadian rhythms - implications for obesity. Endocr Rev 2010;31:1-24. 
-37 Gomez-Abellan P, Madrid JA, Lujan JA, Frutos MD, Gonzalez R, Martinez-Augustin O, de Medina FS, Ordovas JM, Garaulet M: Sexual dimorphism in clock genes expression in human adipose tissue. Obes Surg 2012;22: 105-112.

38 Hernandez-Morante JJ, Gomez-Santos C, Margareto J, Formiguera X, Martinez CM, Gonzalez R, MartinezAugustin O, Madrid JA, Ordovas JM, Garaulet M: Influence of menopause on adipose tissue clock gene genotype and its relationship with metabolic syndrome in morbidly obese women. Age (Dordr) 2011; DOI: 10.1007/s11357-011-9309-2.

-39 Yang L, Li P, Fu S, Calay ES, Hotamisligil GS: Defective hepatic autophagy in obesity promotes ER stress and causes insulin resistance. Cell Metab 2010;11:467-478.

40 Rubinsztein DC, Marino G, Kroemer G: Autophagy and aging. Cell 2011;146:682-695.

-41 Viscomi MT, D’Amelio M: The 'Janus-faced role' of autophagy in neuronal sickness: focus on neurodegeneration. Mol Neurobiol 2012;46:513-521.

-42 Zhou L, Zhang J, Fang Q, Liu M, Liu X, Jia W, Dong LQ, Liu F: Autophagy-mediated insulin receptor downregulation contributes to endoplasmic reticulum stress-induced insulin resistance. Mol Pharmacol 2009; 76:596-603.

-43 Cinti S, Mitchell G, Barbatelli G, Murano I, Ceresi E, Faloia E, Wang S, Fortier M, Greenberg AS, Obin MS: Adipocyte death defines macrophage localization and function in adipose tissue of obese mice and humans. J Lipid Res 2005;46:2347-2355.

44 Denton D, Nicolson S, Kumar S: Cell death by autophagy: facts and apparent artefacts. Cell Death Differ 2012;19:87-95.

-45 Martinez J, Almendinger J, Oberst A, Ness R, Dillon CP, Fitzgerald P, Hengartner MO, Green DR: Microtubuleassociated protein 1 light chain 3 alpha (LC3)-associated phagocy tosis is required for the efficient clearance of dead cells. Proc Natl Acad Sci U S A 2011;108:17396-17401.

-46 Harris J, Hartman M, Roche C, Zeng SG, O’Shea A, Sharp FA, Lambe EM, Creagh EM, Golenbock DT, Tschopp J, Kornfeld H, Fitzgerald KA, Lavelle EC: Autophagy controls IL-1 $\beta$ secretion by targeting Pro-IL-1 $\beta$ for degradation. J Biol Chem 2011;286:9587-9597.

47 Crisan TO, Plantinga TS, van de Veerdonk FL, Farcas MF, Stoffels M, Kullberg BJ, van der Meer JW, Joosten LA, Netea MG: Inflammasome-independent modulation of cytokine response by autophagy in human cells. PloS one 2011;6:e18666.

-48 Roca H, Varsos ZS, Sud S, Craig MJ, Ying C, Pienta KJ: CCL2 and interleukin-6 promote survival of human $\mathrm{CD} 11 \mathrm{~b}+$ peripheral blood mononuclear cells and induce M2-type macrophage polarization. J Biol Chem 2009;284:34342-34354.

-49 Dupont N, Jiang S, Pilli M, Ornatowski W, Bhattacharya D, Deretic V: Autophagy-based unconventional secretory pathway for extracellular delivery of IL-1beta. The EMBO J 2011;30:4701-4711.

50 Zhou L, Liu F: Autophagy: roles in obesity-induced ER stress and adiponectin downregulation in adipocytes. Autophagy 2010;6:1196-1197.

-51 Zhou L, Liu M, Zhang J, Chen H, Dong LQ, Liu F: DsbA-L alleviates endoplasmic reticulum stress-induced adiponectin downregulation. Diabetes 2010;59:2809-2816.

52 Aprahamian TR, Sam F: Adiponectin in cardiovascular inflammation and obesity. Int J Inflam 2011;2011: 376909.

53 Kaushik S, Arias E, Kwon H, Lopez NM, Athonvarangkul D, Sahu S, Schwartz GJ, Pessin JE, Singh R: Loss of autophagy in hypothalamic POMC neurons impairs lipolysis. EMBO Rep 2012;13:258-265.

54 Heckmann BL, Yang X, Zhang X, Liu J: The autophagic inhibitor 3-methyladenine [3-MA] potently stimulates PKA-dependent lipolysis in adipocytes. Br J Pharmacol 2012; doi: 10.1111/j.1476-5381.2012.02110.x.

55 Weidberg H, Shvets E, Elazar Z: Lipophagy: selective catabolism designed for lipids. Dev Cell 2009;16:628630.

56 Ouimet M, Franklin V, Mak E, Liao X, Tabas I, Marcel YL: Autophagy regulates cholesterol efflux from macrophage foam cells via lysosomal acid lipase. Cell Metab 2011;13:655-667.

57 Ouimet M, Marcel YL: Regulation of lipid droplet cholesterol efflux from macrophage foam cells. Arterioscler Thromb Vasc Biol 2012;32:575-581.

58 White E: Deconvoluting the context-dependent role for autophagy in cancer. Nat Rev Cancer 2012;12:401410.

59 Sotelo J, Briceno E, Lopez-Gonzalez MA: Adding chloroquine to conventional treatment for glioblastoma multiforme: a randomized, double-blind, placebo-controlled trial. Ann Intern Med 2006;144:337-343.

60 Amaravadi RK, Lippincott-Schwartz J, Yin XM, Weiss WA, Takebe N, Timmer W, DiPaola RS, Lotze MT, White E: Principles and current strategies for targeting autophagy for cancer treatment. Clin Cancer Res 2011;17: 654-666.

61 White E, DiPaola RS: The double-edged sword of autophagy modulation in cancer. Clin Cancer Res 2009;15: 5308-5316.

-62 Schrijvers DM, De Meyer GR, Martinet W: Autophagy in atherosclerosis: a potential drug target for plaque stabilization. Arterioscler Thromb Vasc Biol 2011;31:2787-2791.

63 Dutta D, Calvani R, Bernabei R, Leeuwenburgh C, Marzett, E: Contribution of impaired mitochondrial autophagy to cardiac aging: mechanisms and therapeutic opportunities. Circ Res 2012;110:1125-1138.

-64 Harris H, Rubinsztein DC: Control of autophagy as a therapy for neurodegenerative disease. Nat Rev Neurol 2012,8,:108-117. 